Voix et Images

\title{
Coup de pistolet dans un concert : Une saison dans la vie d'Emmanuel
}

\section{Henri Mitterand}

Volume 2, numéro 3, avril 1977

Jean Éthier-Blais

URI : https://id.erudit.org/iderudit/200073ar

DOI : https://doi.org/10.7202/200073ar

Aller au sommaire du numéro

Éditeur(s)

Les Presses de l'Université du Québec

ISSN

0318-9201 (imprimé)

1705-933X (numérique)

Découvrir la revue

Citer cet article

Mitterand, H. (1977). Coup de pistolet dans un concert : Une saison dans la vie d'Emmanuel. Voix et Images, 2(3), 407-417. https://doi.org/10.7202/200073ar d'utilisation que vous pouvez consulter en ligne.

https://apropos.erudit.org/fr/usagers/politique-dutilisation/ 


\section{Coup de pistolet dans un concert:} Une saison dans la vie d'Emmanuel

J'accepte par avance le reproche d'outrecuidance, ou de naïveté. Je prétends en effet tenter une lecture' d'Une saison dans la vie d'Emmanuel, alors que je ne suis pas québécois, mais français, que je connais fort mal le roman québécois contemporain, et que je ne sais à peu près rien de l'œuvre - ni de la personne - de Marie-Claire Blais. Beaucoup des informations qu'on pourrait juger indispensables pour ce genre d'exercice me manquent: en particulier une compétence vécue sur l'univers social et mental du Québec au Xxe siècle. J'ignore l'essentiel du Code (culture nationale, rites, pratiques religieuses et sociales, connotations de langage, etc.). Je ne puis même pas procéder de manière contrastive, en comparant le discours de Marie-Claire Blais à celui d'autres écrivains du Québec.

Or, comment repérer le propos idéologique d'un roman, sinon à partir d'un horizon culturel commun au codeur (l'écrivain) et au décodeur? De même, un locuteur natif, de formation scientifique équivalente, sera toujours plus apte qu'un allophone à décrire sa langue. Disons; tout au plus, à ma décharge, qu'en matière de culture et de politique, un observateur extérieur peut parfois plus facilement qu'un "natif " échapper à certains tabous, à certaines pudeurs, à certaines censures. On ne psychanalyse pas volontiers sa mère...

Peut-on parler d'une intrigue, d'une «diégèse», comme on dit maintenant? Non. Tout au plus quelques "tranches de vie», selon la terminologie naturaliste. Et encore, non pas au sens naturaliste de l'expression. Car cette chronique d'une famille du Québec n'est pas située dans le temps historique, ni dans l'espace géographique, comme le souligne Jacques-A. Lamarche ${ }^{2}$. La durée même est insaisissable, partagée entre l'espace d'une saison - les premiers mois de la vie d'Emmanuel - et de 
multiples retours en arrière remontant jusqu'à la venue au monde de Jean le Maigre. Plusieurs points de vue gouvernent la conduite du récit, qui n'est même pas un récit, mais une convergence de discours dont quelques-uns prennent parfois le tour narratif: le point de vue de la grandmère Antoinette, celui de Jean le Maigre, celui d'Emmanuel. Des voix différentes se succèdent ou se croisent, elles-mêmes orchestrées alternativement par l'auteur (à la troisième personne) et par Jean le Maigre (à la première). Le langage n'est pas celui du roman réaliste avec un narrateur extérieur unique - mais celui d'une sorte de geste, ou plus simplement encore d'une parabole familiale, chacun des membres de la famille venant à son tour sur le devant de la scène réciter et commenter l'événement. Encore ne faut-il pas prendre le mot geste dans son sens épique.

A quoi accrocher une exploration des contenus? L'auteur se tait: pas de glose sur l'événement ou sur le personnage, pas de thèse, pas de "morale" ni de conclusion, pas d' «intrusion d'auteur", selon l'expression convenue. Aucune démonstration explicite. Non qu'un discours du texte ne se fasse pas entendre: mais il faut aller le chercher sous chacun des éléments de la structure. Plus que jamais, il faut prendre le mot idéologie dans son sens althussérien (la représentation des rapports imaginaires du sujet aux rapports de production), conduire la recherche en observant les rapports qui relient les sujets de l'énoncé au monde et entre eux, et de là remonter au travail du sujet de l'énonciation, à savoir le sujetécrivain, et peut-être, à travers lui, une classe de sujets. Bien entendu, tout ceci est hypothétique, et doit se garder des clins d'œil avantageux qui caractérisent certaines lectures, prétendument averties des métalangages à la mode de Paris. J'évite donc - qu'on m'en excuse - les grammes, les traces, les disséminations du signifiant, les paragrammes, les phéno- et génotextes... $A$ ce jeu, on manque trop souvent ses sauts périlleux, et j'en connais plus d'un qui s'est noyé piteusement dans des confusions qu'une information linguistique élémentaire lui aurait évitées.

Commençons par la distribution des personnages. On peut les disposer selon une hiérarchie. En tête, la grand-mère Antoinette, personnage central. Elle est, de fait, jusqu'au bout, le principal sujet de l'énoncé. Dans le schéma fonctionnel peu ou prou commun à Souriau et à Propp, elle occuperait vraisemblablement la place du donateur, de l'attributeur. C'est elle qui a dispensé la vie, à l'origine de cette famille; c'est elle qui dispense la nourriture, le nom de baptême, la chaleur, la sécurité - et les sanctions. "Elle leur distribuait avec quelques coups de canne les morceaux de sucre qu'ils attendaient la bouche ouverte." (p. 10) Elle raconte des histoires à Emmanuel, commente à haute voix «les mauvaises nouvelles". De tous les personnages qui participent en acteurs à l'événement, elle seule ne subit aucun processus de dégradation. Pas d'amélioration non plus, au reste. Elle semble là depuis l'éternité, inaltérable; elle « mourra d'immortalité dans un âge avancé». Rien n'est simple, à vrai dire: la grand-mère Antoinette échoue à maintenir autour d'elle la cohésion fami- 
liale. En ce sens, it y aurait tout de même, de la première à la dernière page, une lente dérive négative.

Immédiatement auprès d'elle, Jean le Maigre. Si la grand-mère est la donatrice, lui est une sorte de héros. Héros à la fois malheureux et triomphateur: il mourra, avant la fin, victime du combat pour la lucidité, mais laissant derrière lui la victoire de son texte. II a aussi - ce n'est pas contradictoire avec sa fonction principale - un rôle de donateur. Si Antoinette distribue la nourriture matérielle, Jean le Maigre dispense la poésie. Si Antoinette possède le taire, Jean le Maigre possède le dire. Si la grandmère est la force et le cceur de la famille, Jean le Maigre en est le cerveau, la conscience, la parole - plus que cela: l'écriture. De plus, au palier méta-linguistique, ou, pour mieux dire, méta-diégétique (qui ferait du récit même l'objet d'un échange), Jean le Maigre est l'attributeur: il offre le récit au lecteur, et au Québec la vérité de son être. En quoi il fonctionne à la fois comme sujet de l'énoncé, et comme figure du sujet de l'énonciation, du narrateur.

En arrière d'Antoinette et de Jean le Maigre, au troisième rang d'importance, le Septième et Héloïse; personnages eux-mêmes reparaissant d'un bout à l'autre dụ roman, dont la carrière parcourt plusieurs péripéties, plusieurs tournants, et dont le destin prend valeur de symbole. Le Septième, sorte de faux-héros, compagnon de Jean le Maigre; Héloïse, emblême du calvaire féminin. Un peu plus loin encore, sur l'arbre hiérarchique, les trois autres garçons, Alexis, Pomme, Emmanuel. Et puis, accidentellement évoqués, les autres enfants, notamment les filles, dẹnommées les A: les petites A, Héléna, Maria, et les grandes A, Aurélia, Anita, Anna. Tous destinataires et bénéficiaires du service d'Antoinette, des histoires de Jean le Maigre, ou des niches du Septième. Dans l'environnement immédiat (au moins dans son environnement narratif), le curé, l'institutrice, le Frère Théodule, Mme Octavie, le vieil Horace, et quelques autres que j'oublie.

Encore faut-il nuancer cette sorte d'examen. Tel personnage qui, enfant, apparaît comme un bénéficiaire, plus tard sera investi d'un rôle de donateur, à un échelon secondaire: Héloïse, par exemple, pour ce qui concerne le don du plaisir. Tels personnages qui semblent avoir pour le moins un rôle d'auxiliaires, d'adjuvants, pourraient aussi bien, à un autre palier de l'analyse, se voir définis au contraire comme des forces d'antagonisme, de résistance: c'est un peu le rôle ambigu du curé, qui aide, certes, au départ de Jean le Maigre et d'Héloïse vers la ville, mais qui d'autre part les fourvoie dans une aliénation plus radicale encore que leur misère première. C'est aussi le rôle du père, qui refuse aux enfants le don d'éducation ou à la mère celui de la douceur.

Je répugne à tenter d'algébriser tout cela, comme on le fait parfois en bonne règle sémiotique. Il est clair en tout cas qu'Une saison dans la vie d'Emmanuel n'échappe pas à la pression des schémas canoniques du roman. Chassé par une porte (dans l'éclatement des points de vue et 
la multiplicité des voix, et des voies, narratives), le modèle romanesque rentre par l'autre, avec son petit capital d'archétypes intemporels, venant à la traverse de l'engagement conjoncturel.

Des sous-classes se constituent à l'intérieur de la famille, en intersection mutuelle. La forme manuelle ou non manuelle du travail oppose d'un côté le père, la mère, le Septième, Pomme, et de l'autre Jean le Maigré, Emmanuel, Léopold. Où situer Héloïse? Chez madame Octavie, elle a mis son corps en esclavage; on la rangerait donc dans la première sousclasse. En revanche, par son âge, la grand-mère Antoinette semble se ranger plutôt dans la seconde. Une seconde opposition, celle du maintien à la terre et du départ pour la ville, sépare le père et la mère de quatre de leurs enfants, Jean le Maigre, le Septième, Pomme et Héloïse. Ce sont les plus jeunes qui s'en vont. Les aînés restent avec le père. Quant au trait culture, éducation, il oppose encore le père et la mère, ensemble, à Jean le Maigre. On pourrait aussi tester les personnages au point de vue de leur participation à la marche du récit. On verrait qu'un personnage est plus que les autres exclu de l'aaventure", ou de la "conjointure" (au sens médiéval de ces termes): c'est le père, qui au surplus est fortement péjoré ( "la paresseuse violence de son père») et se voit attribuer un attachement exclusif, régressif, quasi animal, aux besoins élémentaires du corps: manger, fumer, frapper, copuler. Par comparaison, et malgré l'effacement de la mère, "toujours épuisée et sans regard", la femme se trouve valorisée, en raison des rôles assignés à Antoinette et à Héloïse. Aucun de ces personnages, en tout cas, ne se révolte ouvertement contre la donnée familiale, matérielle, et sociale. Chacun s'accommode de la circonstance, au besoin par l'acte clandestin. II n'y a pas ici de meneur... La mise en question n'est pas à chercher dans les postures des sujets représentés (à la différence d'un roman comme Germinal, par exemple), mais dans celle qui conduit le narrateur à inventer, au Québec, en 1965, une telle histoire, résonnant comme aun coup de pistolet" au milieu du concert des géorgiques québécoises ${ }^{3}$.

Deux personnages émergent donc, qui méritent un examen plus attentif: Antoinette et Jean le Maigre. Que l'avatar favori du récit naturaliste soit le récit mythique, on le constate aisément, à suivre les gestes et les mots d'Antoinette. L'âge a conféré à celle-ci la sagesse, la liberté et l'autorité. Elle ne subit plus la loi du mâle. Nourricière et protectrice, abritant sous ses jupes une nichée d'enfants, elle figure à la fois la mère Gigogne, l'Alma mater, la Mère paysanne et la Terre, et aussi la Sainte Mère des litanies: images interchangeables, par le jeu symbolique. "Je suis forte, mon enfant. Tu peux m'abandonner ta vie. Aie confiance en moi. " (p. 8) Elle fédère, elle rassemble, elle maintient le lien familial, de la nais- 
sance à la mort. Elle intègre toutes les valeurs, fait respecter tous les rites: "Une belle tombe, dit grand-mère Antoinette, après un moment de silence - je veux que Jean le Maigre soit fier de moi, jusqu'au bout, une belle mort, dit-elle, avec candeur et humilité, beaucoup de messes pour son âme, beaucoup de fleurs, il aimait tant les cérémonies." (p. 83) Elle raconte les histoires qui unissent le présent au passé, commente les mauvaises nouvelles, et tire les leçons: “Oui, ce sera un beau printemps, disait Grand-mère Antoinette, mais Jean le Maigre ne sera pas avec nous cette année. " (p. 128) Elle dit le regret et l'espérance, dans la même phrase.

Chercherons-nous du sens à tout cela? L'herméneutique a mauvaise presse. On lui oppose la métaphore du texte comme texture, tissu, tissage, tapis tissé, ou encore cercle sans point de départ, ni clôture, ni point d'arrêt du sens. II n'y a pas d'image dans le tapis; seuls des fils qui s'entrecroisent, dans une trame dont on ne peut tirer aucun fil sous peine de la détruire... Mais le moyen de ne pas voir des images dans le tapis? Et de ne pas prêter du sens aux textes? C'est du reste ce que s'empressent de faire les partisans les plus résolus de la "dissémination".

Je verrais donc dans la grand-mère Antoinette la figure, valorisée, d'une sorte de matriarcat rural, que le texte dénote pourtant comme archaïque. Au moment où la famille québécoise traditionnelle éclate, se déchire, mise en péril par la rupture des attaches à la terre, par l'attraction de la ville, par l'extinction de la fécondité, Antoinette exprime la nostalgie d'une structure familiale fortement unitaire. Mais une nostalgie incertaine d'elle-même: Antoinette ne sait plus bien où elle en est. Tout va mal: Héloïse se prostitue, Pomme s'est coupé trois doigts, le Septième est maltraité par l'oncle Laframboise, "à la ville". Tout va bien: Héloïse gagne beaucoup d'argent, le Septième envoie son salaire à la maison, Pomme est bien soigné à l'hôpital. Antoinette pleure, Antoinette rit. Cependant, elle dit à Emmanuel: «Toi aussi tu seras battu si tu poses des questions. Vaut mieux te taire et aller couper du bois, comme les autres." (p. 101) Le Québec est en état de fracture. De quel côté se trouvent la vérité et le bien? Ne seraient-ils pas du côté où reste Antoinette? Elle seule, après tout, garde la confiance, le courage, et, plus encore, le pouvoir d'inspirer l'un et l'autre au dernier-né: “Mais grand-mère Antoinette avait pris Emmanuel dans ses bras, et lui parlait à l'oreille: - Tout va bien; disait Grand-mère Antoinette, il ne faut pas perdre courage. " (p. 128)

Ainsi, seule une aïeule tient encore à la maison des champs, seule elle a souci de ressouder la famille dispersée, seule elle garde le lien avec toute la descendance. La génération intermédiaire n'en a cure. Le père "chasse ses enfants dès qu'ils ne se nourrissent pas tout seuls comme des hommes". (p. 101) Si le. Québec paysan s'allégorise et se valorise dans une vieille femme, qu'en est-il de son avenir? Devant les cahiers de Jean le Maigre, Antoinette se scandalise. Mais aussi elle s'émeut. «Malédiction, oh! Malédiction, s'écriait Grand-mère Antoinette, mais des confidences de Jean le Maigre disparu, de cette âme silencieuse jusqu'au blasphème, elle fortifiait son amour, nourrissait son orgueil." (p. 91) Elle seu- 
le, l'aïeule, la paysanne, est capable de comprendre et de préserver les blasphèmes - et les "prophéties" - de l'écrivain, son petit-fils. N'existerait-il pas une solidarité profonde entre la paysanne du vieux Québec et les intellectuels mal-pensants de 1960, par-delà les mirages et les corruptions de la ville industrielle? Rien n'est simple. Antoinette revendique l'école pour Jean le Maigre, mais c'est celle qui ne coûte rien, et où les enfants "sont bien domptés": le noviciat - d'où Jean le Maigre s'évadera dans la mort.

Jean le Maigre, organiquement et narrativement complémentaire d'Antoinette, protégé par elle au-delà de la mort, est lui aussi un personnage allégorique. Incarnant l'opposition du savoir et de la vie, il reflète le vieux stéréotype populaire, selon lequel l'excès de pensée conduit à la tuberculose, à la méningite - et réciproquement (car ces idées reçues sont réversibles). Symbolise-t-il l'écrivain québécois, comme l'indiquent Michel Brûlé, et Marie-Louise Ollier ${ }^{4}$ ? Soit. A travers Jean le Maigre, la classe intellectuelle du Québec observerait ses propres structures familiales et sociales en transformation. Elle mettrait en question la validité des itinéraires traditionnels et des valeurs imposées. Elle dirait la jouissance du savoir, contre les superstitions. A son père qui menace de lui arracher son livre, Jean le Maigre réplique en montrant "son front blanc": «- II est trop tard, j'ai lu toutes les pages. On ne peut pas brûler les pages que j'ai lues. Elles sont écrites là.» (p. 15) Mais alors cette même classe paraîtrait aussi impuissante à faire déboucher cette prise de conscience et cette mise en question sur autre chose que sur la poésie et sur la mort. Destin tragique en vérité: est-il celui de tous les écrivains québécois, même en 1965 ?

Tout ceci, pourtant, n'épuise pas les échos du personnage de Jean le Maigre. II faut faire un détour par les récurrences thématiques qu'orchestre Une Saison dans la vie d'Emmanuel. Et d'abord, les constantes structurelles de la société d'avant 1960: les servitudes du travail et les misères de la subsistance sur la terre québécoise, quand on a seize enfants et peu d'arpents; le poids de ce que l'idée reçue appelle les fatalités, pour en dissimuler le caractère non inéluctable: la tuberculose, les maternités innombrables, l'ignorance, le suicide; les aliénations de la ville, qui sont d'une autre sorte que celles de la terre, mais non moins asservissantes: le pensionnat religieux, l'usine, le bordel, l'accident, la délinquance; et, contrôlant et alourdissant toute cette misère physique et intellectuelle, l'omniprésence de l'Église. Partout des abbés, partout des Frères, qui se relaient pour conduire les pécheurs au repentir, et les assurer de la miséricorde. Monsieur le curé, le jour des funérailles, mange à la droite de la grand-mère. Autour de la tombe de Jean le Maigre, la voix forte des prêtres recouvre le murmure des petits Frères.

Thèmes et stéréotypes se confondent. Or il y a dans ce roman deux espèces de stéréotypes. Et tout le texte, au fond, met en scène leur conflit. Aux discours et aux rites du catéchisme, s'opposent les gestes et les 
actes que dicte la nature. L'éducation religieuse craque sous les coups de la paillardise. Le plaisir du corps éteint la crainte du péché. La foi exaltée d'Héloïse déguise une tentation qui finit par se satisfaire sans relais, ni délai: «Mon frère et moi, écrit Jean le Maigre, avons été très surpris, et heureux de l'être - en découvrant que notre sœur faisait par elle seule ce que nous aimons faire à deux, ou à quatre, quand Alexis et Pomme sont réveillés, mais ils sont si paresseux qu'ils préfèrent dormir. " (p. 39-40) Seule la paresse peut concurrencer le désir. Le discours de la sensualité submerge allégrement celui de la vertu. Certes, il n'a rien d'original. Mais ses stéréotypes - les enfants masturbateurs, le notaire salace, le mari violeur - se voient attribuer ici un pouvoir sans commune mesure avec celui de l'effort têtu des prêtres pour les maintenir dans la nuit et le silence. Dits, proclamés par le texte de Jean le Maigre, ils transforment le fait en valeur. La confession, l'école, la maison de correction, ne sont que croûtes vides. Les démons l'emportent. L'œuvre de Jean le Maigre s'intitule: "Journal d'un homme à la proie des démons". L'école même flambe. Jean le Maigre descend en enfer, où son frère Léopold, qui s'est pendu le jour du Vendredi Saint, l'a précédé. Mais c'est visiblement le seul chemin de la libération. II faut passer par le mal et la mort pour dépouiller les vieilles servitudes. II faut passer par l'acceptation de tous les désirs qui travaillent et le corps, et la pensée.

Ce qui est donc mis en question ici, c'est d'abord l'organisation de la famille traditionnelle, notamment le pouvoir du père. L'autorité paternelle, instituée en loi absolue par l'institution civile et l'institution religieuse, encourage le viol légal, l'asservissement de la fermme et des enfants. A époux borné et indifférent, famille nombreuse, nichée pléthorique, qui condamne les enfants mâles à la prolétarisation (agricole ou citadine), et les filles à l'esclavage familial, à la réclusion conventuelle, ou à la prostitution.

Désobéir au père n'est qu'un péché parmi tous ceux contre lesquels l'Église met en garde. Le message religieux, par le pouvoir impératif, performatif, dont le revêt la société de référence, aliène littéralement ceux qui l'écoutent: il les rend fous, délirants, schizophrènes, au moins aussi longtemps que la nature, en eux et malgré eux, engendre le péché. Dans la complicité de la nuit, les enfants ne se privent guère de jouer, solitairement ou en groupes. Le matin venu, ils se précipiteront à la confession. Chacun, ainsi, se divise. Seule la prolifération familiale bénit le désir. Celui qui prétend échapper à l'inquisition du dogme n'a d'autre issue que le suicide (Léopold), la mort (Jean le Maigre), ou la dépravation (le Septième). Car le départ pour la ville n'apporte qu'illusion... Ce n'est qu'un autre clivage, un autre éclatement intérieur. D'une certaine façon, la villẹ 
libère. Le père et le prêtre y sont moins proches. Mais la ville aussi désagrège; elle meurtrit, détruit, viole. Et même elle règle, tarifie, institutionnalise la violence et le viol. Pomme, à l'hôpital, Héloïse, à l'Auberge de la Rose publique, ont seulement changé de lois, et de prison. Le Christ entre les images obscènes dénonce une duperie fondamentale, la coexistence pacifique, voire la complicité des deux instances où la femme est à la fois reine et esclave sous le regard et le contrôle de l'homme: l'Église et le bordel. On n'est pas tout à fait loin de Jean Genêt. "Monsieur l'Abbé, dit Madame Octavie, ma charge est aussi grande que la vôtre, vous n'allez pas encore me contredire là-dessus "... (p. 114)

Si ce dispositif de personnages, le destin assigné à chacun d'eux, l'opposition mutuelle des actes, des lieux et des modes de l'action, peuvent se déchiffrer, constatons qu'ils s'efforcent de saccager ironiquement les structures et les valeurs qui sont chères au Québec: à la ville comme à la terre. Cela posé, on n'en est que plus à l'aise pour montrer les limites de ces audaces. Toute littérature de scandale vise à démasquer le refoulé social et politique des idées et des langages reçus, à déplacer les censures qui le cachent. Mais toute littérature de scandale a aussi ses propres censures, son propre refoulé, historiquement explicables.

Les silences qui me frappent le plus sont ceux qui gomment toute allusion à l'argent, au régime de la propriété, aux formes économiques du travail agricole et du travail urbain, aux mécanismes de la rémunération, au fonctionnement des pouvoirs. Ce père et ces fils aînés, «assoupis autour de la table, protégeant leur assiette comme un trésọr », de quoi vivent-ils? Sont-ils journaliers, ou propriétaires de leur terre, et de quelle étendue? Où va la plus-value de leur travail? Silence également, sur les corrélations de cette famille avec les autres unités et les autres niveaux de la stratification sociale. Tout se passe comme si elle vivait en autarcie, n'ayant de rapports qu'avec la terre et avec l'Église. Certes, le père a mené à la ville ses deux "vagabonds", Pomme et le Septième, "comme apprentis dans une manufacture de souliers». Certes, Héloïse a pour clients fidèles des marchands, des médecins, des notaires et des étudiants. Mais ces silhouettes archétypales ne dépassent pas en épaisseur celles des "Physiologies" de l'époque romantique, ou du théâtre de Labiche, en plus lubriques. La mise en scène dérive vers une satire de mœurs déjà vue ailleurs, où le Notaire, le Maire et le Curé suffisent à figurer les satisfactions de l'être bourgeọis. N'est-ce pas un théâtre d'ombres?

D'où quelques questions. La main qui écrit Une saison dans la vie d'Emmanuel, et qui désigne ce qui est devenu intolérable, n'en méconnaîtrait-elle pas les facteurs profonds, ou ne se trouverait-elle pas, au moins momentanément, dans l'incapacité de les inscrire dans le roman? Jean le 
Maigre ne connaît pas le verbe mentir. «Le directeur, pense le Septième, va nous demander de conjuguer le verbe mentir et Jean le Maigre ne le sait pas. $\infty$ ( $p$. 36) Peut-être faut-il aussi comprendre qu'il ne sait pas, au fond, mentir. Mais il ne sait pas non plus porter en tous lieux la lumière. «ll voyait déjà le titre: Poème obscur écrit sur le dos de mon frère pendant son sommeil irréprochable." (p. 37) Une saison dans la vie d'Emmanuel serait-il à l'image des écrits de Jean le Maigre? Non un déplacement radical de la vision critique, mais un "poème obscur", ou, comme dit aussi Jean le Maigre, une «prophétie de famille».

La prophétie est le langage de ceux qui croient au destin - au fatum. Dans les rêves de ses derniers jours, Jean le Maigre ne voit plus "que des fruits pourris dans les branches". "C'est l'hiver partout. II fait froid." (p. 73) Chacun de ses cahiers «trahit une ardeur heureuse et triste». (p. 82) Grand-mère Antoinette peine pour les déchiffrer. Elle peut y lire l'annonce que chacun des enfants suivra fatalement la pente de sa destinée, et la certitude blasphématoire que le malheur eșt d'abord de naître. Emmanuel «aujourd'hui pleure les pleurs amers du berceau» (p. 91). Pomme va vers la prison, le Septième vers l'échafaud, Héloïse vers la luxure salariée. Le passage à la ville n'a rien changé. Quelles que soient les mutations matérielles qui l'affectent, la condition de l'homme est promise au malheur, parce que le mal est dans la nature humaine. Livrés à eux-mêmes dans la liberté de la nuit, les enfants de l'homme deviennent naturellement les insouciants complices du Diable. De là leur familiarité avec le sexe, avec le feu et avec la mort. De là leur satanisme. Et aussi leur puritanisme, par la complaisance que Jean le Maigre met à décrire, én lui et en ses frères, l'instinct de mort mêlé à l'instinct de plaisir.

C'est pourquoi je discuterais volonitiers l'analyse de Lucien Goldmann et de Michel Brûlé, qui laïcisent et optimisent à l'excès la vision de Marie-Claire Blais. "Le mépris et la haine de Marie-Claire Blais pour la société traditionnelle, écrit Lucien Goldmann, n'ont, bien entendu, pas changé d'un roman à l'autre [c'est-à-dire de la Belle Bête à Une saison dans la vie d'Emmanuel]; mais à l'absence d'avenir de la Belle Bête se substitue maintenant la perspective de l'urbanisation, de la création d'une communauté canadienne-française urbaine, qui n'est certes pas un idéal mais a, malgré tout, un caractère positif5. " Tout indique que l'urbanisation n'est pas la libération, mais la substitution d'un système de contraintes à un autre, le changement de décor du Mal. Le pessimisme de Jean le Maigre, Christ qui meurt pour une rédemption improbable de la terre québécoise, ne connaît ni le doute, ni la consolation. Face au jésuitisme des prêtres, qui admettent toujours un accommodement avec le péché pourvu que le rite demeure sauf, Jean le Maigre tient un discours de type janséniste: hors de la grâce, point de salut, et la grâce n'est pas la chose du monde la plus répandue.

A vrai dire, l'optimisme incurable de la grand-mère Antoinette ne vérifie pas davantage le jugement que je viens de citer. Le roman s'achève 
avec l'arrivée du printemps. "Emmanuel n'avait plus froid. Le soleil brillait sur la terre. Une tranquille chaleur coulait dans ses veines, tandis que sa grand-mère le berçait. „ ( $p$. 128) Thème connu. C'est la résurrection, la pâque, germinal... Le fils de l'homme, Jean le Maigre, est mort, mais la vie l'emporte. "Ce sera un beau printemps." Le couple que forment l'aïeule et le nouveau-né symbolise la permanence du Québec dans l'union de ses deux époques, si éloignées l'une de l'autre; et pourtant si unies: le passé et l'avenir. Antoinette a lu les poèmes de Jean le Maigre. Emmanuel ressemble au frère qui vient de mourir: à eux deux, ils assurent le relais. Oui, signifient-ils, l'univers familial, rural, urbain, les structures contingentes de la société québécoise sont inhumaines. Mais la bonté résignée d'Antoinette, autant que le langage lucide de Jean le Maigre, y réintroduisent l'humanité. Le cceur et le langage parlent pour la puissance de I'homme; là résident le salut, l'espoir, la liberté. Au surplus, ils justifient le rôle de l'écrivain, qui conteste et qui lyrise en même temps l'univers de ses aïeux et de ses frères. Sujets majeurs et associés du roman, prophètes contradictoires et complémentaires, Antoinette et Jean le Maigre attestent que l'homme, parce qu'il peut parler son destin (sur les deux portées de la folie - «Son esquise folie», dit de Jean le Maigre le Frère Théodule - et de la résignation), est plus fort que son destin. Image de l'écrivain en abîme dans le roman, Jean le Maigre atteste l'éminente dignité du Verbe. Quoi de mieux pensant?

La lecture "réaliste" et étroitement «sociocritique» d'Une saison dans la vie d'Emmanuel me paraît donc en sous-estimer l'originalité, et faire fausse route. Paradoxalement, la lecture classique, idéaliste, qui y voit un roman “à la gloire de l'homme», affirmant "la conscience de l'être libre $^{6} n$, en repérait avec une intuition plus exacte les phrases idéologiques de base. L'humanisme chrétien, sous ses deux couleurs - la sombre et la claire, l'hivernale et la printanière, la lucidité fiévreuse de Savonarole et l'optimisme patient de la mère de Dieu - colle à ce roman, en dépit de ses furieux sursauts d'anticlérisalisme. Qui y verrait, par une superficielle application du modèle goldmannien ou lukacsien, la mise à nu des contradictions fondamentales du Québec, et l'ouverture vers une «culture nouvelle", pis encore, "une poésie de la société franco-canadienne modernisée et urbanisée ${ }^{7} \%$, se méprendrait et sur sa vraie richesse et sur sa véritable portée historique. Aussitôt après avoir mis à nu les contraintes et les scléroses, le roman de Marie-Claire Blais les allégorise et les naturalise, au lieu de les politiser (au sens profond du terme), donc de les historiser. Oscillant entre la prétendue lucidité du pessimisme et la prétendue lucidité de l'espérance, il s'enferme dans les limites conceptuelles de l'idéologie qu'il semble vouloir faire éclater. C'est en quoi il témoigne historiquement. Bien naîf, tout de même, celui qui trouverait dans les données de cette fable une homologie avec celles du référent social. Sa valeur symptômale est ailleurs: non point primordialement dans son énoncé, mais dans une situation d'énonciation qui mériterait une analyse sociopolitique précise: celle de l'écrivain québécois en 1965, déterminé, quoi 
qu'il en ait, par son appartenance à un type de société où le débat politique demeure refoulé. Une saison dans la vie d'Emmanuel parle pour une époque où la révolution québécoise, pour des raisons d'histoire sociale et culturelle, ne dispose encore ni de forces ni de langages politiques, mais seulement du blasphème. Là est sa vraie grandeur. Modernisation, urbanisation? Ce n'est pas le problème de ce roman! Ni non plus la liberté abstraite du sujet abstrait. Ce qu'il dit, à l'aide du discours même que la culture nationale lui impose en héritage, c'est que ce discours n'est plus possible. Or, rien n'est long comme la mutation des codes idéologiques... C'est le roman du dégel, mais non pas encore celui de la débâcle. Aussi bien, ce qui fait dresser l'oreille au lecteur, ce sont les craquements qui semblent venir d'entre les lignes. Rares sont les œuvres où l'on perçoit ainsi un bruit des profondeurs - ambigu, comme tous les grondements lointains. Ce sont ces œuvres-là qui laissent le lecteur en proie au songe critique. Ce sont les vraies ceuvres réalistes.

Henri Mitterand

Université de Paris - VIII et Université de Toronto

1. J'allais écrire: sociocritique. Mais à quoi bon ces étiquettes ambitieuses. Lisons, tout simplement, et essayons de comprendre... (Une saison dans la vie d'Emmánuel, Montréal, Éditions du Jour, 1965)

2. Jacques-A. Lamarche, "la Thématique de l'aliénation chez Marie-Claire Blais », Cité libre, juillet-août 1966, p. 27-32.

3. Stendhal, le Rouge et le Noir, II, chap. 22: "La politique au milieu des intérêts d'imagination, c'est un coup de pistolet au milieu d'un concert. „

4. Michel Brûlé, "Introduction a l'univers de Marie-Claire Blais", in Sociologie de la littérature, Bruxelles, Éditions de I'Université de Bruxelles, 1973, p. 175 a 185: Marie-Louise Ollier, "Une saison dans la vie d'Emmanuel ", Études francaises, vol. 12, $n^{\circ} 2$, juin 1966, p. 224 a 227.

5. Lucien Goldmann, "Note sur deux romans de Marie-Claire Blais», dans Structures mentales et création culturelle, Paris, UGE, "10-18», 1970, p. 362.

6. Marie-Louise Ollier, op. cit.

7. Lucien Goldmann, op. cit. 